\title{
Libre opinion
}

\section{Afrique : une politique semencière sous influence}

\author{
Danièle Clavel
}

Agronome et généticienne des plantes tropicales, Cirad, UMR Amélioration génétique et adaptation des plantes méditerranéennes et tropicales (Agap), 34398, Montpellier, France

La révolution agricole de l'après-guerre a organisé et déployé la commercialisation en Europe de variétés sélectionnées et homogènes. Cette généralisation fut telle qu'aujourd'hui les paysans européens n'ont plus le droit de disposer comme ils l'entendent des semences de variétés anciennes très diversifiées qu'ils ont sélectionnées au cours du temps. L'Afrique subsaharienne est restée à l'écart de cette intensification agricole que l'on a appelée "révolution verte » en Asie et en Amérique latine. Mais alors que les subventions au secteur agricole ont été drastiquement limitées par les politiques d'ajustement structurel conduites en Afrique par la Banque mondiale et le Fonds monétaire international à partir des années 1970, une agriculture familiale y a perduré en utilisant notamment des variétés-populations végétales du même type que celles qui ont aujourd'hui presque disparu en Europe. Malgré les sécheresses et une forte pression sur le foncier agricole, la production par actif agricole a augmenté de $25 \%$ en moyenne en Afrique sur la période 1961-2001 : les rendements ont crû à un taux annuel moyen de 1,6 \% (Benoit-Cattin et Dorin, 2012).

Aujourd'hui, ces mêmes institutions qui ont négligé l'agriculture, alliées à des multinationales semencières et à des fondations privées très puissantes financièrement, se mobilisent efficacement pour favoriser l'essor rapide d'un marché semencier en Afrique sur le schéma occidental. Dans le modèle agricole promu à partir des années 1950, le fer de lance de la modernisation a été la généralisation de variétés hybrides ou de lignées pures créées pour être performantes dans les conditions de culture artificialisées, c'est-à-dire non limitantes en termes d'eau et d'intrants agricoles (Ahmadi et al., 2013). Cette politique d'intensification a été accompagnée d'un dispositif réglementaire, celui de l'Union des professionnels pour la protection des obtentions végétales (UPOV), taillé sur mesure pour un seul type variétal, celui des lignées et des hybrides dont les génotypes sont fixés, contrairement aux variétés-populations d'antan. Le modèle d'agriculture intensive associé à ces variétés «pures » a fait ses preuves en termes d'augmentation de la productivité mais son coût en termes d'érosion de la biodiversité agricole, de surconsommation énergétique, de pollutions et d'émissions de $\mathrm{CO}_{2}$ devrait logiquement interdire ou limiter ses applications aux pays du Sud.

Le secteur agricole dans les pays africains, amplement dominé par les agricultures familiales, est très largement le premier bassin d'emploi et de revenu de ces pays. C'est pourquoi le dossier actuel du développement et de la réglementation d'un marché semencier est très sensible. Des lois semencières sont en voie de mise en œuvre dans différents pays du continent mais il apparaît que l'on n'ait pas pris la mesure des implications et des risques qu'une réforme aveugle ferait courir à la viabilité des agricultures familiales. Pour cela, la décision des États devrait être éclairée notamment par la recherche publique pour le développement agricole. Ce n'est guère le cas mais certaines alertes commencent à émerger dont cet article se fait l'écho.

Auteur correspondant : clavel@cirad.fr 


\section{Au centre de la création d'un marché africain des semences, la question réglementaire}

La « modernisation variétale et semencière » des trente glorieuses en Europe, selon les termes de Bonneuil et Hochereau (2008) a créé et organisé une offre semencière privée de variétés génétiquement homogènes et traçables. Ce marché est aujourd'hui monopolisé par quelques multinationales semencières ${ }^{1}$, notamment en ce qui concerne les " grandes cultures", les cultures dites secondaires ayant été négligées à la fois par le commerce et par la recherche. On souligne trop peu que les augmentations de rendement obtenues grâce aux nouvelles variétés dans les pays du Nord ont tenu plus au soutien sans faille des pouvoirs publics et de l'ensemble de la recherche agricole sur la durée qu'au prodige de la technologie de la génétique végétale. La même idée de " modernisation variétale et semencière » est reprise pour «nourrir l'Afrique » alors que les variétés paysannes dont les semences sont autoproduites constituent à l'heure actuelle 70 à $80 \%$ des semences échangées par les paysans en Afrique (Louwaars et de Boef, 2012 ; Cirad, 2013). Ce qui a changé dans le contexte mondial depuis les années glorieuses, ce sont les préoccupations liées aux pertes de biodiversité et au changement climatique. Afin de diminuer la pression sur les ressources naturelles et de limiter les pollutions, on reconsidère aujourd'hui l'intérêt des agricultures traditionnelles qui, pendant des siècles, ont fait évoluer leurs systèmes agricoles.

En Afrique, les systèmes vivriers sont généralement économes en intrants, résilients, sources d'agrobiodiversité et de sauvegarde des savoirs écologiques traditionnels locaux (Altieri, 2004). De tels systèmes incluent des variétés-populations anciennes dont la valeur adaptative $^{2}$ est avérée. On sait aujourd'hui que la sélection moderne en fixant les génotypes et en minimisant l'hétérozygotie au niveau paysan limite l'expression de cette adaptabilité (Thomas et al., 2012). Les agriculteurs sélectionnent, échangent ou vendent leurs semences dans des systèmes traditionnels dits informels. Ces systèmes prédominent en Afrique, la sélection, la production de semences et leur diffusion y sont organisées par les paysans eux-mêmes (Louwaars et de Boef, 2012). Ces modes de production de semences sont principalement

\footnotetext{
1 Trois firmes, Monsanto, Pionner/DuPont et Syngenta, concentrent $44 \%$ du marché (Ducos et al., 2013).

2 La valeur adaptative fait principalement référence à la notion de "plasticité phénotypique » en réponse aux contraintes environnementales. Cette faculté d'adaptation est présente dans les variétés-populations anciennes du fait de leur composition génétique non fixée, de leur évolution-sélection et d'une certaine permanence dans un milieu donné lui-même en évolution.
}

vivriers et très diversifiés selon les espèces, les espaces et les contextes socioculturels (Clavel et al., 2014). L'industrie semencière, au contraire, aujourd'hui bien implantée en Afrique comme en témoignent les communications de Bayer CropScience $^{3}$, Dow AgroSciences ${ }^{4}$ et l'Alliance for $^{\prime}$ Food Sovereignty in Africa $\left(\right.$ AFSAFRICA ${ }^{5}$ ), promeut un système formel, donc un cadre réglementaire. Il s'agit du modèle marchand conventionnel dans lequel les variétés, généralement de type lignées, hybrides et OGM, sont homogènes et génétiquement identifiables de façon à sécuriser les droits de propriété intellectuelle et à favoriser l'innovation brevetable. Dans cette optique, la protection commerciale d'une population qui se reproduit ellemême dans les champs paysans en se modifiant au cours des générations et en se mélangeant au gré d'associations variétales à géométrie variable est impossible. L'aspect fixé du génotype est donc une donnée-clé. C'est pourquoi la question réglementaire sur les droits de propriété et les normes autorisant la commercialisation est indissociable de celle du type variétal à promouvoir.

Actuellement, les pays africains francophones, membres de l'Organisation africaine de la protection intellectuelle (OAPI), ont déjà adopté le système de protection des obtentions végétales de l'UPOV 1991 (le système européen actuel) et les autres États africains sont tous engagés dans cette voie. L'accord de l'OMC sur les aspects des droits de propriété intellectuelle touchant au commerce (ADPIC) de 1994 prévoit que les États ayant adhéré à l'UPOV aient la possibilité de mettre en place un système sui generis (de leur choix) pour la protection des variétés végétales. Le groupe africain à l'OMC $(\mathrm{OMC}$, 2003) avait observé qu'un juste équilibre devrait être trouvé entre les droits des communautés locales, des agriculteurs et des sélectionneurs, en tenant compte du Traité international sur les ressources phytogénétiques pour l'alimentation et l'agriculture (TIRPAA) (FAO, 2009) et de la loi type africaine sur la biosécurité (African Union, 2001) ; par ailleurs, certains États au sein du conseil des ADPIC avaient défendu la convention UPOV de 1978 comme système sui generis.

Cependant, le dispositif de l'UPOV 1991 étant le seul acte auquel les États peuvent aujourd'hui adhérer, la préférence pour celui de l'UPOV 1978 est fermée à la signature pour les États non membres de l'UPOV avant 1991, ce qui est le cas de pratiquement tous les pays africains. Le système UPOV 1991 préserve les droits d'utilisation des ressources génétiques à des fins de recherche mais, contrairement à l'UPOV 1978, il restreint les droits des

\footnotetext{
3 http://www.cropscience.bayer.com/Magazine/HealthyFood-for-All.aspx.

4 http://agri4africa.com/index.php?dirname=docs_02news letters /00010newsletter.php\&lookupArticleId=242.

5 http://afsafrica.org/fr/acquisition-of-africas-seedco-bymonsanto-groupe-limagrain-neo-colonial-occupation-of-africas-seed-systems /.
} 
paysans en interdisant toute utilisation de semences autoproduites en dehors de leurs propres champs. Selon ce système, pour échanger ou commercialiser des semences et plants, il faut que les variétés soient inscrites dans un catalogue officiel des espèces et variétés (semences certifiées). Or, l'inscription au catalogue n'est légale que si la variété satisfait notamment aux normes de «Distinction, Homogénéité, Stabilité » (normes DHS). Dans les pays du Nord, les lignées et hybrides sont généralisés (à l'exception notable des semences du Réseau Semences Paysannes) mais, en Afrique, la majeure partie des variétés vivrières utilisées par les agriculteurs sont des variétés-populations (ou variétés-composites, en référence au fait qu'une variété est constituée de plusieurs génotypes). Les règlements semenciers calqués sur le système UPOV 1991 ne peuvent donc pas être appliqués tels quels tant que la production semencière demeurera informelle et portera sur des variétés de type population. C'est le cas, par exemple, du règlement de l'espace CEDEAO (Communauté économique des États de l'Afrique de l'Ouest) (CEDEAO, 2008) que le Conseil ouest et centre africain pour la recherche et le développement agricoles (CORAF) a pour mission de promouvoir avec les financements de 1'United States Agency for International Development (USAID) et de la Banque mondiale.

Les semences des variétés-populations paysannes africaines sont autoproduites dans des systèmes traditionnels de production et d'échanges de semences. Si l'intérêt de ces variétés locales a été clairement reconnu par les institutions internationales et de recherche depuis la Convention sur la diversité biologique (CDB), les systèmes traditionnels dans lesquels ces variétés sont produites et diffusées ne sont pas reconnus légalement et sont méconnus des chercheurs du fait de la prégnance du «paradigme industriel de la variété pure et stable » (Bonneuil et Hochereau, 2008). Ainsi, la Banque mondiale, qui alloue une grande partie des financements de la recherche et du développement agricoles en Afrique, développe depuis 2007 en Afrique de l'Ouest le Programme de productivité agricole en Afrique de l'Ouest (PPAAO/WAAPP). Le PPAAO accueille et coordonne ses activités avec celles du Programme Semences de $1^{\prime}$ Afrique de $1^{\prime}$ Ouest (PSAO/WASP ${ }^{6}$ ) financé par 1'USAID (9 millions de dollars, 2013-2017) qui mobilise les chercheurs nationaux, notamment pour la mise au point de cadres juridiques calqués sur les réglementations du Nord, sans action dirigée sur la reconnaissance des systèmes informels existants, pour l'instant (Wekundah, 2012).

L'enjeu central dans le processus d'adhésion des États africains au système de 1'UPOV 1991 réside donc dans la capacité de négociation d'une révision de ces normes

\footnotetext{
6 http://www.usaid.gov/west-africa-regional/fact-sheets / west-african-seed-program-wasp.
}

DHS pour tenir compte des réalités écologiques, économiques et sociales. Parmi ces réalités, figure l'existence de systèmes informels efficaces de production de semences s'exerçant sur des variétés-populations adaptées aux conditions de culture, aux pratiques et utilisations locales et dont la diversité génétique est irremplaçable. Si l'intérêt évident de tels systèmes n'est pas reconnu et étudié, ces variétés pourraient être rendues illégales pour la commercialisation (l'échange à titre non onéreux restant permis), il s'agirait donc bien d'une révolution semencière aux conséquences néfastes comme le déplore l'African Centre for Biosafety qui annonce dans son rapport (2012) « une recette pour un désastre ».

\section{Le credo de la fondation Bill et Melinda Gates}

Créée en 2000, la Fondation Bill et Melinda Gates est la première fondation au monde par son capital et ses capacités de financement. Elle est le premier bailleur de fonds pour les recherches sur la santé et sur l'agriculture et l'alimentation en Afrique. Pour le développement agricole, l'accent est mis sur l'Afrique subsaharienne et l'Asie du Sud, en prônant "l'accès à des semences plus résistantes ${ }^{7}$ » comme condition centrale d'une augmentation de la productivité agricole durable pour les petits exploitants.

La puissance financière de la Fondation Gates associée à des alliances stratégiques (par exemple, des exmembres de la Banque mondiale font partie du conseil d'administration ; la politique d'investissement est judicieuse, notamment par l'acquisition en 2008 d'actions Monsanto pour un montant de 20 millions d'euros), pèse très lourd, en particulier sur le système de prise de décisions des États africains. Un prix mondial, le World Food Price, créé par Norman Borlaug, le père de la "révolution verte », vient asseoir la légitimation scientifique. Ce n'est pas l'équivalent du « Nobel pour l'agriculture », comme la presse a coutume de le présenter car il est sponsorisé par des organismes privés, la Fondation Rockefeller et la Fondation Gates ainsi que Pioneer, Monsanto (5 millions de dollars en 2008) et Syngenta. Il couronne annuellement des personnalités aussi différentes que Mohamad Yunus (1994), Lula (2011) mais aussi le directeur de la technologie de Monsanto $\left(2013^{8}\right)$. L'argument central développé par la Fondation Gates porte sur l'intérêt pour l'Afrique de disposer des dernières avancées technologiques en termes de variétés, option présentée comme la clé de hauts rendements,

\footnotetext{
7 http:/ /www.gatesfoundation.org/fr/What-We-Do/GlobalDevelopment/Agricultural-Development.

8 https://www.worldfoodprize.org/index.cfm/24667/ 24410/three_biotechnology_scientists_awarded_2013_world_ food_prize.
} 
quelles que soient les conditions. Le rôle des autres facteurs de production dans l'élaboration du rendement est à peine mentionné. Les hautes valeurs adaptatives mises en évidence dans les variétés locales ainsi que les connaissances associées aux ressources génétiques soulignées par le TIRPAA ne sont pas évoquées. La simplification du message est un gage d'efficacité dans les plaidoyers politiques que la Fondation Gates inclut dans ses objectifs stratégiques.

L'Alliance pour une révolution verte en Afrique (Agra), créée en 2006, regroupe les fondations Gates, Rockefeller et Syngenta, la Banque mondiale dans son ensemble, des membres influents de grandes universités américaines, comme Cornell et Iowa State, et l'USAID. Koffi Annan fut le premier président du conseil d'administration de l'Agra, certains membres de la FAO sont un soutien actif de l'alliance. L'Agra, à travers le Program for Africa's Seed System (PASS ${ }^{9}$ ), met en œuvre un plan d'action pour la création d'un marché de semences africain qui présente l'avantage rare de la sécurisation des financements. Une autre fondation dont l'objectif est de promouvoir l'offre privée des semences en Afrique, l'African Agricultural Technology Foundation (AATF) existe depuis 2003. Créée dans le but de faciliter les partenariats public-privé, on y retrouve les fondations Rockefeller, Gates et Syngenta. L'AATF et l'International Maize and Wheat Improvement Center (CIMMYT), qui intervient de longue date sur le maïs en Afrique de l'Est et du Sud, sont liés à travers notamment le programme Water Efficient Maize for Africa ${ }^{10}$ sur un financement de la Fondation Gates et dans lequel on retrouve Monsanto parmi les partenaires.

Ce réseau d'alliances est positionné sur une logique économique d'offre semencière dans laquelle le dispositif de formation de jeunes sélectionneurs africains s'intègre harmonieusement. Depuis 2008, en effet, de jeunes Africains sont formés à l'Université du Ghana, où le nouveau Centre d'Afrique de l'Ouest pour l'amélioration des plantes (en anglais, West Africa Centre for Crop Improvement, $\left.\mathrm{WACCI}^{11}\right)$, financé par l'Agra, est installé. L'objectif déclaré sur le site web du WACCI est « d'améliorer la sécurité alimentaire en Afrique grâce à une approche centrée sur le développement de variétés "supérieures" par des sélectionneurs africains formés par le centre utilisant les connaissances conventionnelles et modernes ». Les étudiants travaillent sur différentes plantes cultivées en Afrique avec toutefois une prédilection pour le maïs $(28 \%)$, choix dont on sait qu'il renforce l'option réglementaire (Louwaars et de Boef, 2012). Ce projet est doté d'un financement évalué à 15 millions de dollars (75 milliards de CFA) pour les 8 premières années. Il n'y a pas d'équivalent en Afrique dans la recherche publique et la coopération internationale. La puissance financière et les alliances facilitées par l'héritage commun de Borlaug s'interalimentent, permettant au WACCI de mettre en place très rapidement des accords de coopérations avec les centres CGIAR comme AfricaRice, basé au Bénin, ou l'International Institute of Tropical Agriculture (IITA), basé au Nigeria.

Le réseau tissé par la Fondation Gates en Afrique confère à l'industrie semencière privée, principalement américaine, un rôle décisif dans la définition des axes de recherche et de développement agricole des États. Cette vision unique ciblée sur les semences améliorées, certifiées et diffusées en top-down détourne de la prise en compte de systèmes agroécologiques diversifiés encore présents en Afrique et que l'on tente de réalimenter dans les pays du Nord. Un devoir de vigilance et de responsabilité s'impose donc aux scientifiques. Cependant la recherche de financements, une spécialisation de plus en plus poussée, une certaine méconnaissance des enjeux de développement agricole mais aussi de l'agronomie entravent l'exercice. L'implication de la communauté scientifique agricole pour le développement des pays du Sud est encore trop faible face aux enjeux semenciers qui, non éclairés par la sphère publique, risquent de reproduire en Afrique un modèle aujourd'hui fortement remis en cause dans les pays du Nord en raison de ses effets environnementaux.

Néanmoins, on assiste à une prise de conscience très récente qu'il convient de saluer et de soutenir. Le concept développé par Louwaars et de Boef (2012), Integrated Seed Sector Development (ISSD), vient d'être entériné par l'Union africaine et un projet pilote d'une durée de 2 ans (financé par la Fondation Gates) démarre dans deux pays (Éthiopie et Ouganda ${ }^{12}$ ). Le concept central de cette opération pilote est de prendre en compte la diversité des systèmes semenciers existants, notamment en étudiant ceux dits informels (donc en reconnaissant leur importance). Cette initiative récente est modeste mais montre qu'il est possible d'infléchir une politique jusque-là monolithique.

\section{Références}

African Centre for Biosafety, 2012. Harmonisation of Africa's seeds laws: a recipe for disaster. Players, motives and dynamics, The African Centre for Biosafety, http:/ /acbio.org.za/harmonisation-of-africas-seeds-laws-a-recipe-for-disaster/.

African Union, 2001. African model law on safety in biotechnology, African Union, http://hrst.au.int/en/biosafety/modellaw.

Ahmadi N., Bertrand B., Glaszmann J.C., 2013. Rethinking plant breeding, in Hainzelin E. (Ed.), Cultivating biodiversity to transform agriculture, Springer, 91-140.

\footnotetext{
12 http://www.issdseed.org/issd-africa-about-project.
}

\footnotetext{
9 http://archive.agra.org/what-we-do/seed/.

10 http://wema.aatf-africa.org/project-brief.

11 http://www.wacci.edu.gh/.
} 
Altieri M.A., 2004. Linking ecologists and traditional farmers in the search for sustainable agriculture, Frontiers in Ecology and the Environment, 2, 1, 35-42.

Benoit-Cattin M., Dorin B., 2012. Disponible alimentaire et productivité agricole en Afrique subsaharienne, Cahiers Agricultures, 21, 5, 337-347.

Bonneuil C., Hochereau F., 2008. Gouverner le «progrès génétique ». Biopolitique et métrologie de la construction d'un standard variétal dans la France agricole d'aprèsguerre, Annales. Histoire, Sciences Sociales, 6, 1305-1340.

Ducos G., Pujol J.-L., Teillant A., 2013. Pour un secteur des semences diversifié et innnovant, Note d'analyse du Commissariat général à la stratégie et à la prospective, 10/2013, 5, http://www.strategie.gouv.fr/publications/un-secteursemences-diversifie-innovant.

CEDEAO (Communauté économique des États de l'Afrique de l'Ouest), 2008. Règlement c/reg.4/05/2008 portant harmonisation des règles régissant le contrôle de qualité, la certification et la commercialisation des semences végétales et plants dans l'espace CEDEAO, 17-18 mai, http://www.reca-niger.org/IMG/ pdf/Reglements_Semences_CEDEAO_2008.pdf.

Cirad, 2013. Gérer collectivement la biodiversité agricole en Afrique, Valorisation et innovation en partenariat, 24, janvier, http://www.cirad.fr/var/cirad/storage/original/ application/18b46b74ad049e02337fd52ac5c74786.pdf.

Clavel D., Bazile D., Bertrand B., Sounigo O., vom Brocke K., Trouche G, 2014. Biodiversité agricole et systèmes paysans de production de semences, in Sourisseau J.-M. (Ed.), Agricultures familiales et mondes à venir, Versailles, Quæ/ AFD, 287-302.

FAO, 2009. Traité international sur les ressources phytogénétiques pour l'alimentation et l'agriculture (TIRPAA), FAO, http:/ / www.planttreaty.org/fr/content/textes-du-trait\%C3\%A9versions-officielles.

Louwaars N., de Boef W.S., 2012. Integrated seed sector development in Africa: a conceptual framework for creating coherence between practices, programs, and policies, Journal of Crop Improvement, 26, 39-59.

OMC, 2003. Comment faire progresser l'examen de l'article 27:3 b) de l'accord sur les ADPIC ? Communication conjointe du Groupe africain à la Réunion du Conseil des aspects des droits de propriété intellectuelle qui touchent au commerce, Organisation mondiale du commerce, 4-5 juin, OMC Doc IP/C/W/404.

Thomas M., Demeulenaere E., Dawson J.C., Khan A.R, Galic N., Jouanne-Pin N., Remoue C., Bonneuil C., Goldringer I., 2012. On-farm dynamic management of genetic diversity: the impact of seed diffusions and seed saving practices on a population-variety of bread wheat, Evolutionary Applications, 5, 8, 779-795.

Wekundah J., 2012. Why informal seed sector is important for food security, African Technology Policy Studies Special Papers Series, 43, http://www.atpsnet.org/Files/sps43.pdf. 